\title{
Wireless Sensor Networks for Rehabilitation Applications: Challenges and Opportunities
}

\author{
Abdelkrim Hadjidj $^{\mathrm{a}}$, Marion Souil ${ }^{\mathrm{a}}$, Abdelmadjid Bouabdallah ${ }^{\mathrm{a}}$, Yacine Challal ${ }^{\mathrm{a}}$, Henry Owen $^{\mathrm{b}}$ \\ ${ }^{a}$ Université de Technologie de Compiègne. Heudiasyc, UMR CNRS 6599, France \\ ${ }^{b}$ Georgia Institute of Technology, School of Electrical and Computer Engineering, Atlanta, Georgia 30332-0250 USA
}

\begin{abstract}
Rehabilitation supervision has emerged as a new application of wireless sensor networks (WSN), with unique communication, signal processing and hardware design requirements. It is a broad and complex interdisciplinary research area on which more than one hundred papers have been published by several research communities (electronics, bio-mechanical, control and computer science). In this paper, we present WSN for rehabilitation supervision with a focus on key scientific and technical challenges that have been solved as well as interdisciplinary challenges that are still open. We thoroughly review existing projects conducted by several research communities involved in this exciting field. Furthermore, we discuss the open research issues and give directions for future research works. Our aim is to gather information that encourage engineers, clinicians and computer scientists to work together in this field to tackle the arising challenges.
\end{abstract}

Keywords: Wireless Sensor Networks, Rehabilitation Supervision, Human Motion Tracking, Healthcare.

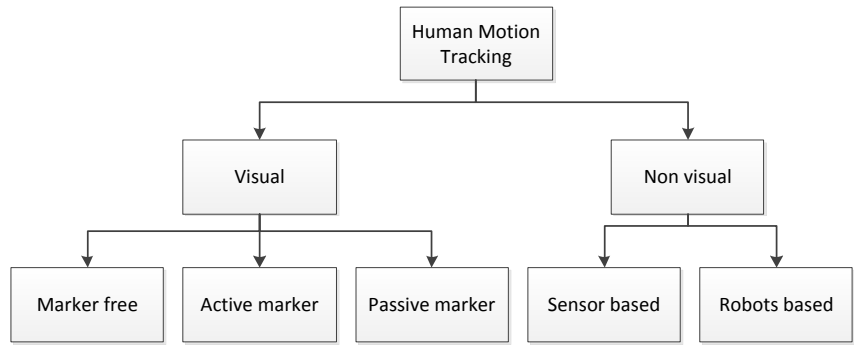

Figure 1: Taxonomy of human motion tracking systems

\section{Introduction}

Rehabilitation is a therapy where the patient performs various physical exercises and activities to achieve a physical functioning level that allows him to return to his initial motor capabilities after an accident, a stroke or a surgery. Studies show that intensive rehabilitation decreases the recovery time and achieves optimal rehabilitation outcomes [1]. Furthermore, physical therapists should continuously monitor and rectify patients during rehabilitation to avoid improperly exercising. Continuous supervision of patients during long term rehabilitation therapy increases the load for physical therapists and medical staff and cost too much for patients. At a time of such challenges, new solutions arise from the need to develop effective, low-cost and easy to use rehabilitation supervision systems suitable for ambulatory or home settings.

Human Motion Tracking systems attracted significant interest in the last two decades due to their potential in rehabilitation

Email addresses: ahadjidj@utc.fr (Abdelkrim Hadjidj), msouil@utc.fr (Marion Souil), bouabdal@utc.fr (Abdelmadjid Bouabdallah), ychallal@utc.fr (Yacine Challal), henry. owen@ece.gatech.edu (Henry Owen) supervision [2]. Several human motion tracking systems have been proposed both in industry and academic research. They can be classified as either visual or non visual systems as depicted in figure 1. Visual human motion tracking systems [3] conduct 3D localization of the patient's body and limbs by combining data of several cameras recording the patient from different perspectives. Marker-free systems [4] track the boundaries of human body while marker-based systems, such as CODA[5] or Qualisys[6], track either light reflective markers (passive markers) or light-emitting diodes (active markers) attached to the patient. Such systems have shown promising performance in rehabilitation supervision due to their accurate localization (error around $1 \mathrm{~mm}[2]$ ). However, they are expensive, invasive and suffer from occlusion and line-of-sight problems.

In robot-based solutions [7], the patient installs his limbs on a robot to perform several movement patterns. The robot moves, guides or just disturbs the movement of the limb while measuring kinematic values such as velocity, acceleration and force [8]. Such systems are recommended for patients with severe disabilities which make them unable to perform exercises by themselves. However, they are expensive, cumbersome and cannot be used in ambulatory or home rehabilitation settings.

Sensors have been used in motion tracking [9] to avoid problems inherent to visual systems such as occlusion and line-ofsight problems [2]. In sensor based systems, the patient wears several small nodes able to assess human movement without interfering with his natural behaviors. These nodes form a network which unobtrusively gathers information regarding position, motion, direction and physiological state. As depicted in Figure 2, a node is composed of several sensors for data collection, a microcontroller with memory for data processing, a radio transceiver for data transmission and a battery for powering all circuits in the device. Using sensors dramatically reduces the 


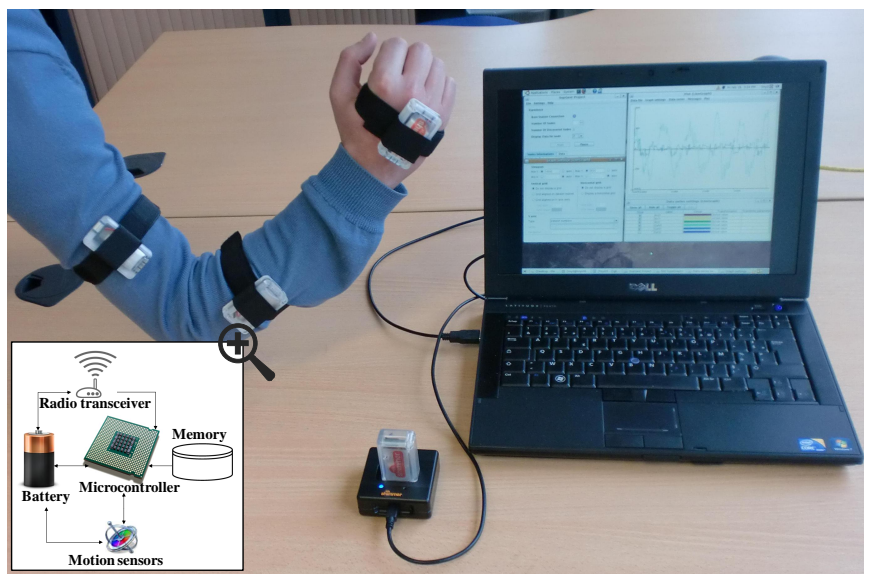

Figure 2: Node architecture: This picture shows a prototype of WSN for rehabilitation that we have developed in a previous project [10]. It is consisting of a set of motion sensor nodes which measure acceleration and angular velocity of each segment of the supervised arm. Collected data is then sent to a central station for data storage and processing

cost and size of rehabilitation supervision systems and opens new opportunities.

Researchers from biomedical, biomechanical and computer science communities have been working toward the development of wireless sensor networks that bring a wave of breakthroughs in providing rehabilitation. Many teams have successfully developed working systems and early clinical results have been already obtained. Indeed, wireless sensor networks have been used in several rehabilitation applications such as stroke rehabilitation, balance training, parkinson's disease and telerehabilitation. The excitement for this technology is motivated by the several benefits associated to long-term monitoring, low cost, rapid deployment, self organization and flexibility features of WSN.

In this paper, we present WSN for rehabilitation supervision with focus on key scientific and technical challenges and design considerations. We thoroughly survey, analyze and discuss works conducted by several research communities involved in this exciting field, namely, electronics, bio-mechanical and computer science communities. Our aim is to gather information that encourage engineers, clinicians and computer scientists to work together in this field. Furthermore, we present interdisciplinary challenges and issues that are still open and give potential directions for future research works.

The remainder of this paper is organized as follows: In section 2, we present related works. In section 3, we propose a classification of different clinical applications of wireless sensor networks for rehabilitation supervision and we present their benefits and their intrinsic characteristics. In section 4, we discuss the design considerations and the challenges that govern these systems. In section 5, we survey existing works on wireless sensor networks for rehabilitation. In section 6 , we address open research challenges and give directions for future works. Finally, we conclude the paper in section 7.

\section{Related works}

There are several surveys covering human motion tracking systems for rehabilitation [2]. These surveys reviewed existing vision-based human motion tracking systems [3] as well as the possible applications of sensors in human posture and movement analysis [9]. However, none of them has considered wireless sensor network technology.

Authors in [11], reviewed wearable bio-sensors for health monitoring. Similarly, Hao et al. [12] provided a comprehensive review of development in wireless sensor networks for human physiological monitoring. A recent work [13] has described several promising WSN healthcare applications and highlighted the key technical challenges that WSN face in healthcare. Almedar et al. [14] organized existing WSN for healthcare into five categories: activity of daily living monitoring, fall and movement detection, location tracking, medication intake monitoring and medical status monitoring. All these works covered a wide range of healthcare applications but they did not consider WSN for rehabilitation.

Rehabilitation supervision may seem as a special case of healthcare supervision. However, traditional WSN for healthcare do not tackle the specific challenges associated with the intrinsic application requirements of rehabilitation (see section 3.3). In fact, sensor diversification, multi-sensor data fusion, realtime feedback for patients and virtual reality integration are examples of features that make WSN for rehabilitation a specific research area with unique hardware, software, communication and architecture design considerations. A recent review paper [15] summarized clinical applications of wearable technology in the general field of rehabilitation. Applications described in this review paper include health and wellness, safety, home rehabilitation, assessment of treatment efficacy and early detection of disorders. However, authors presented works currently undergoing assessment without studying previous works. Furthermore, they did not present the scientific and technical challenges of rehabilitation supervision.

Body Area Networks (BAN) is a specific support of WSN for healthcare applications. Although there are several recent surveys on BAN [16][17][18][19], they are mainly focused on hardware and physical layer (antennas, body-network interferences, signal propagation modeling).

The contributions of our work are manifolds. First, we present WSN for rehabilitation supervision and explain the unique characteristics that differentiate it from legacy WSN for healthcare. Second, we identify the design considerations and the challenges that govern these systems. Third, we review existing works on WSN for rehabilitation and discuss their design objectives. Fourth, we identify open research issues and give several directions for future works.

\section{Wireless Sensor Networks for Rehabilitation}

WSN for rehabilitation have been used in several clinical applications. In this section, we present these different applications and propose a corresponding taxonomy. Also, we highlight the benefits that this technology bring. Finally, we de- 


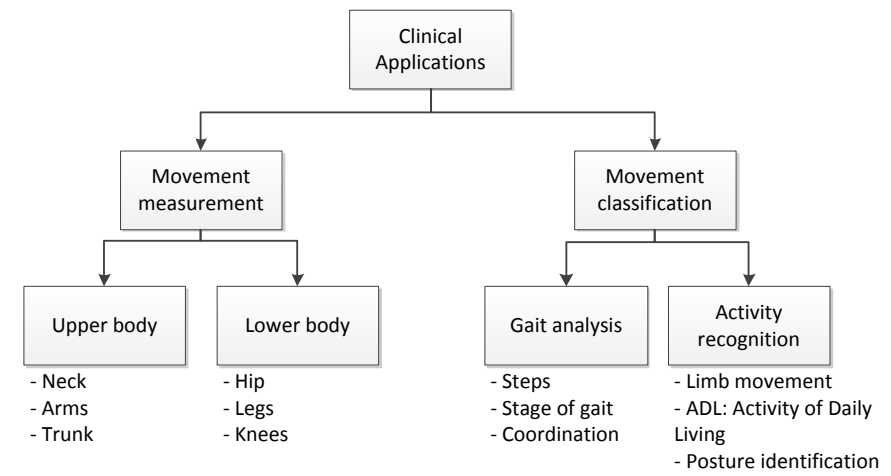

Figure 3: Taxonomy of WSN clinical rehabilitation applications

scribe unique characteristics that make WSN for rehabilitation different from traditional WSN for healthcare.

\subsection{Classification of clinical applications}

The main goal of WSN for rehabilitation is to capture movements and postures of patients for monitoring his motor activities during rehabilitation therapy. Possible clinical applications include cognitive rehabilitation such as cognitive impairment or brain injury treatments, as well as motor rehabilitation such as post-stroke rehabilitation, post-surgery rehabilitation, postaccident rehabilitation or post-disease rehabilitation.

Classifying WSN for rehabilitation is a tough task because of the important number of existing works and the wide range of covered applications. A classification following the clinical applications, like traditionally presented in review papers, is not interesting in rehabilitation case. Indeed, the same architecture can be successfully used in different rehabilitation applications. To avoid such a meaningless taxonomy, we classified existing solutions from a process point of view and we divided them into two main classes that we illustrate in figure 3 . The first class includes WSN designed to measure the patient's movement and activity. In this category, data is continuously collected and recorded in order to be analyzed by specialists. The recorded data can be raw sensors data such as acceleration and velocity or processed data such as limb joints, angles and positions. The second class includes WSN designed to classify the patient movements. In this category, the system extracts features from the collected data and matches them to well known motor patterns in order to answer to the question: "what is the action being performed by the user?".

WSN for upper body movement measurement have been mainly developed for arms and trunk supervision. In [20], authors proposed a network of six motion sensor nodes to track the upper body movement in 3D space at real time. These sensors collect kinematic data in order to measure elbow, shoulder and waist right/left joints. These measured joints were used to animate a 3D human upper body model consisting of a trunk and two limbs. In [21], authors defined a kinematic model to accurately track 3D upper limb motion in rehabilitation of brain injury patients. Furthermore, they developed a methodology based on sensor nodes for calculating six degree of freedom, namely, orientation (roll, pitch and yaw), acceleration, angular velocity and magnetic north.

WSN has also been used to monitor the movement of lower body parts such as knees, legs and hip. The Telefonica's I+D Rehabitic project [22] is a knee telerehabilitation project that offer real time 3D animation of the movement being performed by the patient. Rehabitic fusions data from heterogeneous sensors (accelerometer, gyroscope and magnetometer) to achieve accurate 3D localization of monitored foot. The HipGuard project [23] is a wireless sensor network for patient recovery from hip surgery. HipGuard is based on a set of wireless modules with accelerometer, magnetic and capacitive insole sensors to measure hip/leg position and rotation as well as the force between the foot and the shoe.

Gait analysis is an emerging application of WSN for rehabilitation. Hanson et al. [24] used a wireless sensor node for shuffle/normal gait classification. They proposed a signal processing algorithm for data normalization, features extraction and wavelets pre-processing. Many studies used a wireless sensor network to identify several limb injuries such as osseous, muscular, degenerative and nervous injuries [22]. Ying et al. [25] developed a WSN for detecting steps and respiration phases during treatment of Parkinson's Disease patients. Furthermore, they quantified the degree of the locomotion-respiration coordination using the statistic Kuiper's test [26] in order to determine the stage of the disease and to re-learn coordination.

Activity of Daily Living (ADL) recognition is a promising rehabilitation application. Guraliuc et. al [27] presented a WSN for arm rehabilitation movements detection. They implemented an algorithm based on the RSSI (Received Signal Strength Indicator) to identify and classify the rehabilitation activities. They used a max-win voting strategy to classify seven features derived from the RSSI and to characterize the patient movement. Alhamid et al. [28] used a 3 axis-accelerometer sensor network attached to different body segments in order to identify the patient posture (walking, standing, sitting, lying down or falling). They applied a K-Nearest Neighbor (KNN)[29] classifier to distinguish between features computed by each sensor separately. Later, they merged the different sensors/classifiers data using a Bayesian network [30] in order to identify the patient activity.

Besides motion monitoring, WSN have been used as a research tool for assessment of treatment efficacy, comparison of different treatment protocols, evaluation of recovery and identification of pathological postures and movements. Furthermore, WSN can be used as a support system to improve the efficiency of the rehabilitation process. For instance, the Ubiquitous Rehabilitation Center [31] uses a WSN to manage all aspects of a rehabilitation center. It allows specialists to assign prescriptions to patients, to configure rehabilitation machines for patient's particular prescription (ie. setting resistance, duration and frequency) and to record patient activity while they are carrying out their prescriptions. Bravo et al. [32] proposed a mobile system to monitor frailty of elderly people during rehabilitation. Their solution computes a frailty index based on balance, mobility, endurance and physical activity of the patient and triggers an alarm if it reaches a defined threshold. 
Table 1: Comparison between visual motion tracking and WSN based solution for rehabilitation supervision

\begin{tabular}{lcc}
\hline & Visual motion tracking & WSN based solutions \\
\hline Cost & High & Low \\
Accuracy & High & Good \\
Complexity & High & Low \\
Automation & Moderate & High \\
Feedback & High & Moderate \\
Mobility & Low & High \\
Comfort & High & Good \\
Multi modality & NA & High \\
\hline
\end{tabular}

\subsection{Benefits}

WSN and visual motion tracking systems have different characteristics that we summarize in Table 1. Consequently, using WSN for rehabilitation supervision brings the following benefits:

\subsubsection{Cost effectiveness:}

Health care cost has been rising for several years and will continue to rise even more rapidly in the future. From a cost perspective, the key challenge today is to move healthcare from where it is expensive (hospitals, clinics ) to where it costs less (patient's home). This situation is more challenging for rehabilitation because it is a long-term time consuming therapy. Wireless sensor networks, a low cost technology, is then a key to the successful implementation of telerehabilitation services which can replace the traditional face-to-face services and hence improve the cost effectiveness. Moreover, they reduce the stress on rehabilitation providers as well as improve the quality of life for patients.

\subsubsection{Installation easiness:}

Traditional rehabilitation supervision solutions have been proven effective but limited to well equipped laboratories because of their cost and complexity. For example, using a camera-based monitoring system requires installing several cameras to cover different perspectives like illustrated in figure 4. In addition, these cameras need to be calibrated and correlated to make possible an accurate 3D localization. This example clearly demonstrates that such system is complicated and cannot be used at home by non-technical and a fortiori by disabled persons. Wireless sensor networks for rehabilitation enable the development of user-friendly easy to use monitoring solutions. Indeed, WSN are easily deployable systems where the patient wears several sensors and starts data collection through a simple user interface. WSN are often based on frameworks that support service discovery, dynamic network construction and configuration as well as sensor node management. All these features improve the system usability and make it suitable for everyday use.

\subsubsection{Care access improvement:}

Restoration of functions is a slow process that requires prolonged courses and regular visits to rehabilitation providers. Commuting to a hospital is expensive and troublesome because

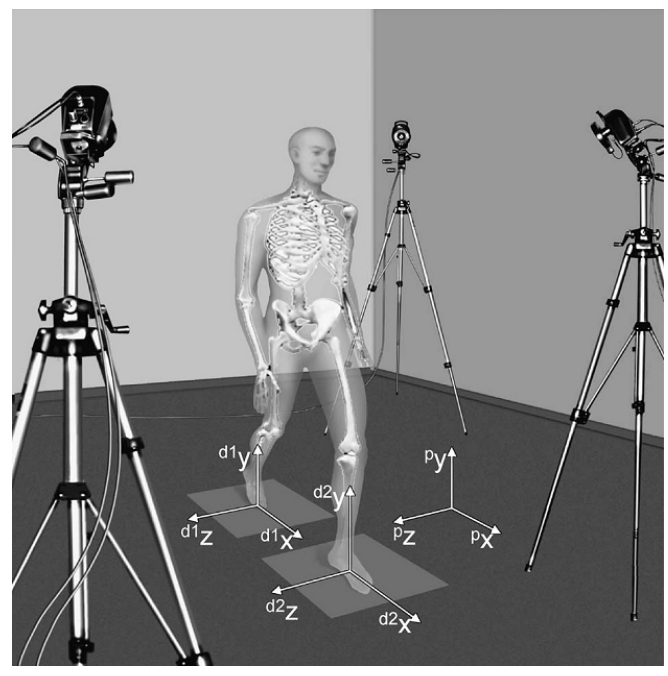

Figure 4: Camera-based monitoring system [33]

of patient disabilities and inadequate transportation. In addition, the patient often needs another person to bring him to the therapy center. Consequently, tele-rehabilitation from a remote location is important for the patient. Wireless sensor networks are ideal for developing at-home rehabilitation solutions able to save time and travel overhead for both patients and care providers. Indeed, the patient can perform their exercises at home and receive automatic real time feedback from the monitoring system. Furthermore, recording and sending patient's sessions to the hospital gives the therapists more data for deeper analysis and feedback.

\subsubsection{Patient mobility:}

One major benefit of wireless sensor networks for rehabilitation is extended mobility. Namely, lack of wires, small form factor and light weight sensor nodes enable patients to move freely while performing their exercises. A typical scenario of mobility is a patient using a wireless sensor network to do his exercises in a public park. Data is collected at the patient's mobile and relayed to the hospital databases through a $3 \mathrm{G}$ connection.

\subsubsection{Increasing motivation:}

Psychological aspect of rehabilitation is of a primary importance. Patients, often affected by post event depression, need to be motivated and encouraged to adhere to long term medical therapies. Rehabilitation exercises are usually considered boring for patients because of their repetitive nature. Wireless sensor networks coupled to other technologies such as video gaming [34] and virtual reality [35][36] are able to tackle the lack of motivation problem. An interesting system would be a combination of a WSN and a serious game, incorporating both pedagogical and entertainment elements, with increasingly difficult levels to make the patient feel the challenge and to increase its involvement in the rehabilitation process. 


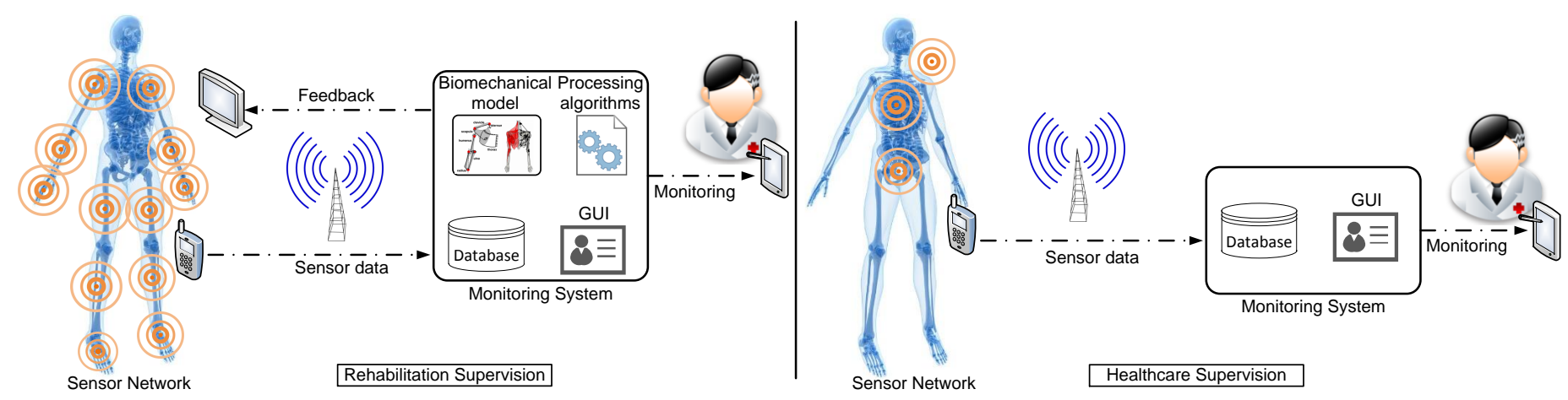

Figure 5: Rehabilitation Supervision VS Healthcare Supervision

\subsubsection{Multi-modal sensing:}

People concerned by rehabilitation are mainly elderly, poststroke or post-surgery patients who may have a critical health status. Consequently, the global physiological state of these high risk patients needs to be monitored during rehabilitation sessions. Wireless sensor networks have the capacity to monitor different vital signals in addition to motion signals. They enable the use of heterogeneous sensors on a common architecture. This feature leads to the development of multi-modal WSN for safe rehabilitation supervision. A wireless sensor network for rehabilitation can incorporate an ECG (Electrocardiogram sensor) to monitor heart activity of weak heart patient to avoid over-exercising during rehabilitation sessions. This solution can generate an alarm when the heart rate of the patient reaches a threshold to avoid heart attacks.

\subsection{Specificities}

WSN for rehabilitation may seem as a special case of healthcare supervision. However, traditional WSN for healthcare do not tackle the unique challenges associated with rehabilitation. In typical medical applications of WSN, a single sensor node is used per vital parameter. Furthermore, positions of sensors are not of a primary importance and can be chosen approximatively. Since limbs are composed of segments jointed together, several sensor nodes are required to monitor the same limb during rehabilitation which increase the number of used nodes. These nodes are carefully placed and accurately localized. Furthermore, they measure several signals (acceleration, angular velocity, muscle activation ...) to capture different characteristics of the movement (displacements, positions, joint angles ...). With several nodes monitoring the same limb, information from one node become highly correlated with other nodes information. Thus, multi-sensor data fusion is used to combine information from different nodes and sensors to produce clinically relevant information.

Another key difference between legacy healthcare supervision and rehabilitation is feedback delivery. In healthcare supervision, the information collected by the sensor network is only used by caregivers to monitor the patient health at realtime [37]. In rehabilitation supervision, the information is used by both caregivers and patients. On one hand, doctors view the summative kinematic data to assess the progress of the patient and adjust the therapy accordingly. On the other hand, the collected data is processed and provided as feedback to the patient to allow him to adjust his movements at realtime. Feedback can have different forms such as on-screen 3D avatar showing the patient's movement compared to the correct movement. Virtual reality and video games are also emerging as a new visual feedback technics specific to rehabilitation.

Unique characteristics of rehabilitation supervision have an impact on the hardware, software and communication protocol design. First of all, the architecture of WSN for rehabilitation is different as we illustrate in figure 5. At the software layer, new services are introduced for multi-sensor data processing and fusion as well as for realtime feedback generation. To enable the extraction of high level information, sensor sampling is performed at high frequency which generates a large amount of data. The high rate data exchanged at realtime between the components of the system have an impact on the communication protocol design. In brief, although challenges faced by legacy WSN for healthcare supervision are in many ways similar to rehabilitation supervision, there are intrinsic differences between the two which create new challenges or make traditional challenges harder to tackle as we show in the next section.

\section{Challenges of WSN for rehabilitation}

WSN can bring a wave of breakthroughs in rehabilitation supervision. Nevertheless, several scientific and technical challenges have to be tackled before fully enjoying the power of this technology. In this section, we present the design considerations and challenges that must be tackled in the design of new WSN for rehabilitation.

\subsection{Design considerations}

Applications of WSN are numerous and from one application to another, the design considerations may be different. In order to provide a better understanding of the current research challenges in WSN for rehabilitation, we have considered the requirements and the characteristics of rehabilitation supervision and then we have derived either new challenges specific to this application or traditional challenges that become harder to tackle because of unique needs of rehabilitation. At the top 


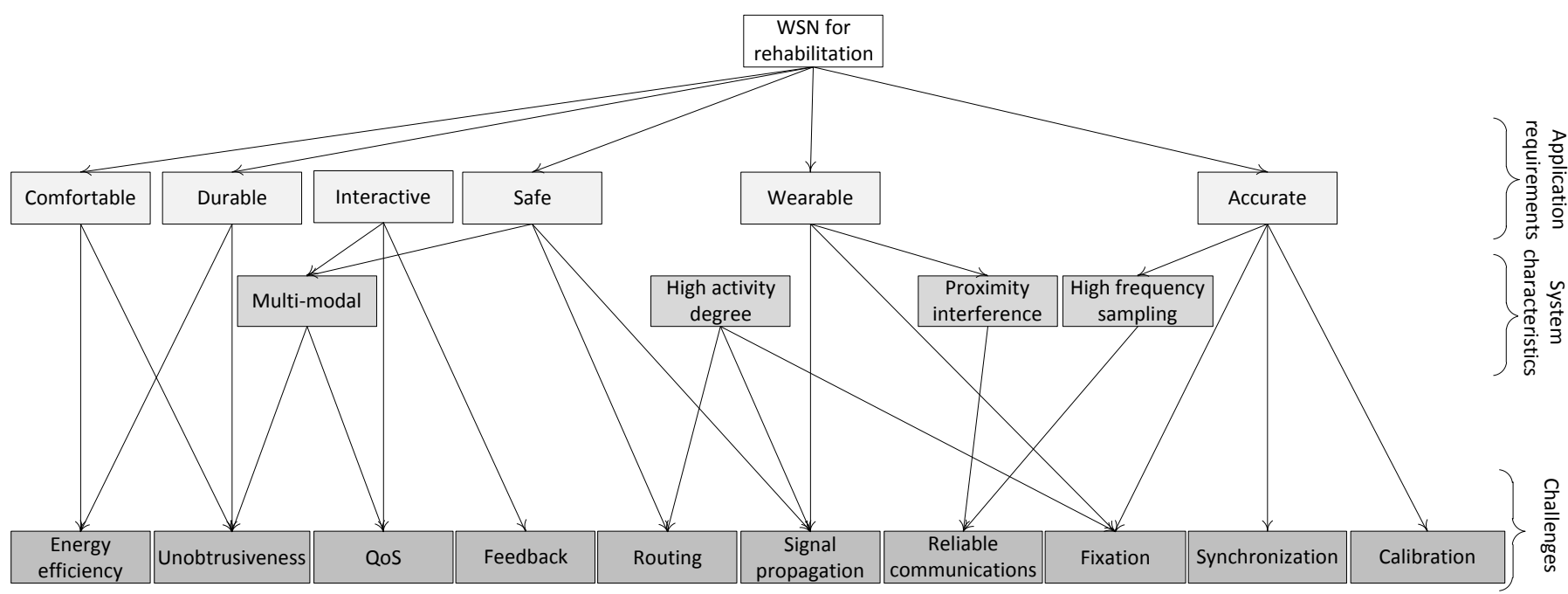

Figure 6: Requirements and challenges of WSN in rehabilitation supervision

level of figure 6 , we present the requirements of rehabilitation supervision from the application perspective. At the next level, we enumerate the characteristics from system perspective. These characteristics are consequences of a one or several design requirements. At the third level, we show the challenges resulting from combining one or several characteristics and requirements:

- Wearability: WSN for rehabilitation must be carried by the patient during exercising. Consequently, sensor nodes must have an attachment to ensure a steady fixation even during rude movements. Taking into account the proximity of node on the body, interferences should be considered at network layers to provide reliable communications.

- Comfort: since the patient carries several sensor nodes for long periods and during physical activities, the comfort become an important design consideration to provide the highest degree of convenience. This requires the development of unobtrusive devices and small form factor batteries.

- Durability: sensor nodes should have a maximum lifetime without battery charging which may be burdensome especially for elderly or impaired patients.

- Accuracy: likewise WSN for healthcare, the system must show high accuracy in data collection and data processing in order to extract correct medical information. For the same reason, sensors should be sampled at high frequencies to correctly get the phenomenon being monitored.

- Safety: considering the criticality of the application, the proposed solution should be safe for the patient, namely, it should be context aware to consider the patient environment and physiological state. Also, it should be without any adverse or harmful effect on the patient.

- Interactivity: to replace the traditional clinical rehabilitation, WSN based solutions must be interactive to guide the patient during his therapy. Particularly, the sensor network must provide realtime feedback such as indicating correct or incorrect movements to allow the patient to adjust his movements immediately.

\subsection{Challenges}

The combination of the application requirements and the system characteristics creates the following challenges:

\subsubsection{Unobtrusiveness}

In rehabilitation supervision, the patient carries several sensors attached on his body during rehabilitation sessions. Since he performs physical exercises for long periods, unobtrusiveness becomes one of the basic needs and requirements for the acceptance of this technology. Nodes must be lightweight and small enough to provide the highest degree of comfort and convenience [38]. As previously discussed, multimodal supervision enables safe rehabilitation monitoring of high-risk patients. However, integrating different types of sensors into one device makes it a challenge to build small multimodal sensor nodes. Furthermore, the battery size constitutes the biggest miniaturization bottleneck since it is the largest contributor to the sensor size and weight. Indeed, developing smaller batteries with fair energy resources is a hard challenge since the battery capacity is directly related to its size [39].

\subsubsection{Fixation}

One potential direction to improve the accuracy of collected data during rehabilitation is the development of new node attachment techniques. If a sensor node does not have a good contact with skin, it may drift from its initial position and influence the quality of data. This issue is a challenge because of the high-degree activity of the patient. Indeed, several papers have reported that skin mounted accelerometers may overestimate amplitudes and low-pass filter the acceleration signal [40][41]. Harms et al. [42] indicated critical aspects that should 
be considered in posture and garment selection when developing comfortable garments for rehabilitation. Finally, we should mention that the proposed attachment technique should be easy to handle even for people with significant loss of functions [2].

\subsubsection{Energy efficiency}

Energy efficiency is a significant challenge in rehabilitation supervision because of the specific characteristics of the application. Indeed, the high rate sampling of sensors and the transmission of the high amount of generated data consume much of energy. To achieve low power consumption, sensor nodes often use low duty cycling by going off most of the time. Unfortunately, duty cycling can not be used in rehabilitation supervision because motion sensors must be continuously sampled. Nodes battery charging is burdensome for elderly and disabled rehabilitation patients. In order to prolong the sensor node life time and reduce charging overhead, the battery volume must be considerable. However, this goes against the unobtrusiveness requirement. Consequently, development of novel power supplies is paramount. It is widely recognized that wireless communication is the most power consuming process in wireless sensor networks. Energy aware MAC protocols [43] must be developed to control the dominant sources of energy waste: idle listening overhearing and collision [19]. At the same time, rehabilitation applications need highly reliable communications and high data rates, resulting in contradictory interests in the network design. Trade-offs must be found to conciliate performance and network lifetime which is a hard challenge.

\subsubsection{Body impact on signal propagation}

In rehabilitation supervision, sensor nodes are worn by the patient and the effect of human body on the wireless transmission must be understood. The signal propagation through the body is affected by the diffraction around the body and the reflection from the body [16] which affects the communication performance especially if sensors are placed on different side of the body [44] which is often the case in rehabilitation. Consequently, we need accurate and realistic propagation models in order to optimize or to develop new communication device and protocols. Developing propagation models is a key challenge because of the complexity of body structure (skin, bone, liquid) and especially because of the high degree of activity during rehabilitation exercises. Indeed, Franco et al. [45] showed that even small involuntary movements along with respiration can cause significant signal fading. Beside the effect of human body on the wireless transmission, the adverse biological effect of radio transmissions on human body must be investigated too.

\subsubsection{Reliability}

In rehabilitation supervision, several sensor nodes must be used to measure the different signals characterizing the movement of the monitored limb. For instance, three nodes at least are required to monitor the movement of an arm (fixed on the hand, on the segment between the shoulder and the elbow and on the segment between the elbow and wrist). Also, monitored signals, such as acceleration and velocity, are continuous and
Table 2: Sensors commonly employed in rehabilitation

\begin{tabular}{lcccc}
\hline & Data rate & Bandwidth & Sampling & Data \\
\hline Acc & High & $35 \mathrm{kbps}$ & $0-500 \mathrm{~Hz}$ & 12 bits \\
Gyro & High & $35 \mathrm{kbps}$ & $0-500 \mathrm{~Hz}$ & 12 bits \\
ECG (12 leads) & High & $288 \mathrm{kbps}$ & $100-1000 \mathrm{~Hz}$ & 12 bits \\
ECG (6 leads) & High & $71 \mathrm{kbps}$ & $100-500 \mathrm{~Hz}$ & 12 bits \\
EEG (12 leads) & High & $43 \mathrm{kbps}$ & $0-150 \mathrm{~Hz}$ & 12 bits \\
EMG & Very high & $320 \mathrm{kbps}$ & $0-10000 \mathrm{~Hz}$ & 16 bits \\
Temperature & Very low & $120 \mathrm{bps}$ & $0-1 \mathrm{~Hz}$ & 8 bits \\
Image sensor & Very high & $1 \mathrm{Mbps}$ & - & - \\
Video sensor & Very high & $100 \mathrm{kbps}$ & - & - \\
\hline
\end{tabular}

the amount of collected data needs to be large enough to correctly get the phenomenon being monitored. Proximity issues regarding the placement of several nodes on the body and the continuous streaming of high volumes of data lead to heavy collisions and extra energy consumption. Developing a MAC protocol which provides reliable data transfer is a key challenge for rehabilitation supervision. The proposed protocol must achieve maximum throughput in spite of severe interference and relatively low bandwidth available in WSN.

\subsubsection{Quality of Service}

Considering the limited buffer size and the high sampling rate, the communication protocol should support online communications to send data before being dropped out due to buffer overflow [46]. Moreover, the communication protocol should support realtime communications with guaranteed delays to deliver realtime feedback to the patient during rehabilitation sessions. As the health of patients is involved, the system must guarantee the delivery of alerts, such as fall of an elderly during exercising, within strict delay constraints. For this purpose, emergency data prioritization mechanisms should be developed. WSN for rehabilitation supervision often combine several sensors which we summarized in table 2 . This table clearly shows that generated traffic have different characteristics (data rate, sampling frequency, packet size). Due to specific characteristics of each traffic type, the communication protocols should implement service differentiation to differentiate between packets and to process them accordingly.

\subsubsection{Routing}

Star topology with one-hop communications has been assumed to be the best network topology for body area networks considering the traffic pattern and the short distance between nodes. However, with unique characteristics of rehabilitation and the impact of human body on wireless transmissions, one can justifiably ask if really one-hop communications are better than multi-hop communications in terms of reliability and energy efficiency. To hop or not to hop data in WSN for rehabilitation is a non trivial question that must be considered. The work presented in [47] was the first attempt to point the benefits of using multi-hop communications in wireless body area networks (WBAN). Later, Natarajan et al. experimentally investigated the impact of network topology on the average number of retransmissions, network lifetime [48] and packet delivery ratio [49]. They concluded that the multi-hop strategy is more 
reliable especially in outdoor settings. This can be explained by the fact that wireless communication through the human body is impossible even at high power transmission.

\subsubsection{Calibration}

Inertial sensors (accelerometer and gyroscope) are the most used sensors in rehabilitation supervision. Unfortunately, these sensors suffer from errors in motion estimation because of measurement noise and fluctuation of offsets. This problem is known as the "drift problem" [50] and should be solved by calibration techniques. Sensor calibration is an adjustment applied to the sensor output so it matches the real value of the monitored signal. This adjustment is made by applying an offset to the measured value in order to eliminate a constant component called "fixed error" as well as a variable component called "random error" which may vary over time and environment (eg. electrical noise, thermal drift). Building calibration techniques that take into consideration the fluctuation of the random error when the sensor environment changes (eg. ambient temperature) is a challenge that must be tackled.

\subsubsection{Synchronization}

In WSN for rehabilitation, data generated at different sensor nodes is highly correlated. Indeed, several nodes are usually fixed on the same limb at different segments to monitor its movements. The sensed signals must be matched following their sampling instant to enable the reconstruction of the correct original movement of limb. Furthermore, sensor nodes do not share a common clock and need a synchronization mechanism to time stamp their data with the same reference. Consequently, accurate data time stamping and tight synchronization are critical problems that need to be addressed.

\subsubsection{Feedback}

Providing useful and motivating feedback to patients is both a key factor in the motor recovery process and a real challenge to address. Indeed, indicating incorrect movements and immediately correcting them have been shown to optimize motor recovery [51]. In addition, feedback have been described as more interesting and enjoyable, thereby encouraging the patient to spend more time in therapy [52]. The main challenge in providing feedback is to convert raw sensor data into relevant clinical information. This requires the development of efficient signal processing algorithms and novel kinematic/kinetic models. For instance, the conversion of angular velocity and accelerations into displacement and positions (particularly specifying the reference point) is an active research area. Multi-sensor data fusion is also a problem since several sensors are needed for clinical data extraction. Overall, these algorithms must be realtime and guarantee response within strict time constraints to preserve the interactivity and reinforce the benefits of therapy. Finally, there is a clear need for adequate feedback presentation. For example, the feedback should be multimodal (image, sound, movement, touch) to be useful for patients with different impaired functions such as visual or hearing problems. Virtual reality and interactive gaming are emerging as a new presentation approaches for the patient movements and progress.

\section{Review of wireless sensor networks architectures for Re- habilitation}

In this section, we review conducted projects on WSN for rehabilitation in order to highlight addressed challenges and proposed solutions. We classify research works, with respect to the design objectives, into four main classes : sensor node design, communication protocols design, signal processing design and frameworks design.

\subsection{Sensor node design}

To achieve comfortable and non-invasive continuous rehabilitation supervision, sensor nodes should satisfy the following requirements: minimal weight, miniature form factor, wearability and unobtrusiveness. However, these requirements represent a challenging task for sensor node design since the batteries capacity is directly related to its size. Accordingly, sensor nodes should implement low power consumption hardware to enable long life time with small batteries. In addition, wireless medical sensor design should promote flexibility and easy integration of other sensors/actuators.

In [53], authors designed a small sensor unit with gyroscope and accelerometer sensors to build a simplified, low cost and wearable sensor network for gait evaluation. In this study, authors focussed on measurement of ankle and knee joint angle during gait rehabilitation. In order to reduce error accumulation inherent to inertial sensors and unknown initial joint angles, they applied a stationary Kalman filter to the difference between inclination measured by the accelerometer and the gyroscope. Although the form factor of the developed sensors is small, they are interconnected by wires which may limit the patient's activity and level of comfort and thus negatively influence the measured results.

Lim et al. in [54] have developed a wireless sensor network which is used in upper arm rehabilitation for stroke patients. Their work focussed on the design of a wearable, compact and low cost sensing module able to measure the arm movement with accuracy. The wearable sensor module consists of an Optical Linear Encoder (OLE), an accelerometer sensor, a 16 bits microcontroller and a CC2420 radio-transceiver. The wearability of the sensor module is done with a plaster that adheres to the human skin as illustrated in figure 7.

Actis (Activity Sensor) described in [55] and [56] consists of a standard Telos mote and a custom Intelligent Signal Processing Module (ISPM) board. The authors extended the Telos mote capabilities by adding two perpendicular dual axis accelerometers and a bio-amplifier for Electrocardiograph (ECG) sensor. This sensor is integrated into a multi-tier telemedicine architecture for computer-assisted physical rehabilitation application.

The iNode presented in [57] is a miniaturized sensor node with a Force Sensitive Sensor (FSS) and a Respiratory Inactive Plethysmography (RIP) sensors. iNode measures the locomotive and respiratory signals and analyzes the coordination between them in order to facilitate the rehabilitation for Parkinson patients. It implements an on-board 2-phases steps detection algorithm and a respiratory phase detection method, which have been optimized for the MSP430 processor hardware. 
Table 3: Comparison of sensor node design projects

\begin{tabular}{cclcccc}
\hline Ref & New platform & Integrated sensors & Fixation & Wireless & Energy & Onboard processing \\
\hline \hline 53] & $\checkmark$ & $\begin{array}{l}\text {-Uniaxial gyroscope } \\
\text {-3 axis accelerometer }\end{array}$ & $\times$ & $\times$ & $\times$ & $\begin{array}{l}\text {-Error and offset reduction } \\
\text {-Lower limb joint angle measurement }\end{array}$ \\
\hline$[54]$ & $\checkmark$ & -OLE & $\checkmark$ & $\checkmark$ & $\times$ & -Upper limb joint angle measurement \\
\hline$[55]$ & $X$ & $\begin{array}{l}\text {-2 axis accelerometer } \\
\text {-ECG }\end{array}$ & $\times$ & $\checkmark$ & $\checkmark$ & $\begin{array}{l}\text {-Acceleration filtering } \\
\text {-User activity estimation }\end{array}$ \\
\hline [57] & $\checkmark$ & $\begin{array}{l}\text {-FSS } \\
\text {-RIP }\end{array}$ & $X$ & $\checkmark$ & $X$ & $\begin{array}{l}\text {-2 phases step detection optimized } \\
\text { for the MSP430 }\end{array}$ \\
\hline \hline
\end{tabular}

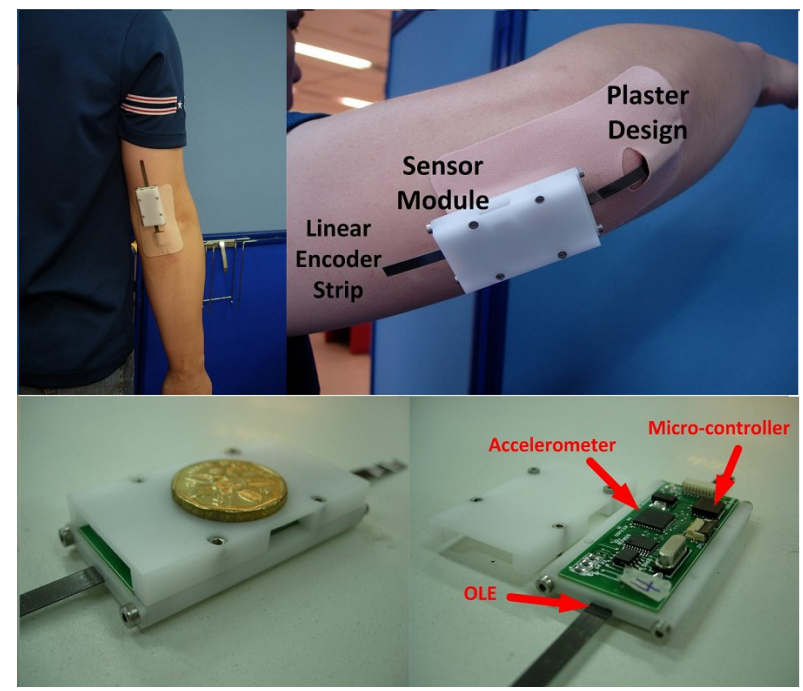

Figure 7: Plaster design of sensor module [54]

\subsection{Communication protocol design}

WSN for rehabilitation have two specific characteristics which impact the network performance and cannot be ignored. First, the placement of several adjacent sensor nodes on the body causes serious interferences problems. Second, sensor nodes need to continuously stream high volumes of data at a determined rate over long periods in order to extract clinically relevant information. Consequently, reliable communication protocols able to handle large amount of data, in spite of severe interference and relatively low bandwidth, are needed.

Some existing works address the challenges of designing a communication protocol for wireless sensor networks used in rehabilitation supervision. In [58], authors developed a communication protocol based on the 802.15.4 standard to network the iNode sensors. However, it does not support a mesh topology and has a communication range limited to 10 meters. In [59], authors developed a complete telerehabilitation system that combines information from a wearable sensor network and a video conferencing system. The sensor network consists of MicaZ modules with different sensor sets (respiratory belt, instrumented shoes, pulse oximeter and accelerometer). The objective of this work was to study the impact of the number of sensor nodes used and the sampling rate on the reliability of the radio transmission. Authors showed that high sensor sampling frequency dramatically reduces the reliability of the communi- cation protocol but did not propose a solution for this issue.

HipGuard [23] is a wireless sensor network for patient recovery from hip surgery. It is a set of wireless modules with accelerometer, magnetic and capacitive insole sensors to measure hip/leg position and rotation as well as the force between the foot and the shoe. Authors used ANT [60] networking technology to collect data from sensor modules to the base station. However, this technology presents significant drawbacks such as limited communication range and fixed packet size.

In [61], authors presented an inertial/magnetic wireless sensor network for motion capture which allows monitoring of several patients at the same time. This system has a modular and portable architecture and implements a modified version of the LPRT MAC protocol [62] for data transport. Unfortunately, this protocol showed limited performance because it is implemented on the top of another protocol.

We have proposed in a previous work [46] an hybrid CSMACA/TDMA protocol for WSN for high-fidelity rehabilitation supervision. Our protocol defines two traffic classes which are HRP (High Rate Periodic) traffic sent with TDMA and LRA (Low Rate Aperiodic) traffic sent with CSMA-CA. Although our protocol achieves high performance by combining different medium access techniques, it defines only two traffic classes.

\subsection{Signal processing algorithms}

The development of efficient signal processing algorithms able to handle a high amount of sensor data is of great importance. These algorithms must treat data and provide a real time useful feedback. This is one of the hardest challenges in wireless sensor networks for rehabilitation. Single sensor data is often not sufficient to extract clinically-relevant information. In these cases, more signals have to be combined to produce meaningful information that evolve into medical knowledge.

In [63], authors proposed a wearable sensor system that measures reaction force in order to detect the eight different gait phases during rehabilitation as illustrated in Figure 8. This solution is based on a motion analysis system and a gait phase detection algorithm. To deal with sensor errors and calibration, authors implemented an intelligent calibration process that fuses accelerometer and gyroscope signals.

Zhou et al. [64] designed an arm movement tracking system for rehabilitation of stroke patients and a kinematical model that integrates human arm movements. The system is based on a wearable wired sensor network and a weighted least squares filtering method in order to capture arm movements in 3-D space 
Table 4: Comparison of communication protocols

\begin{tabular}{cccccc}
\hline Ref & $\begin{array}{c}\text { Access } \\
\text { technique }\end{array}$ & $\begin{array}{c}\text { Energy } \\
\text { saving }\end{array}$ & $\begin{array}{c}\text { Service } \\
\text { differentiation }\end{array}$ & $\begin{array}{c}\text { Traffic } \\
\text { prioritization }\end{array}$ & Topology \\
\hline \hline$[59]$ & CSMA & $X$ & $X$ & $X$ & Star \\
\hline$[23]$ & TDMA & $\checkmark$ & $X$ & $X$ & Star \\
\hline$[61]$ & TDMA & $X$ & $X$ & $X$ & Star \\
\hline$[46]$ & CSMA/TDMA & $\checkmark$ & $\checkmark$ & $X$ & Star \\
\hline \hline
\end{tabular}

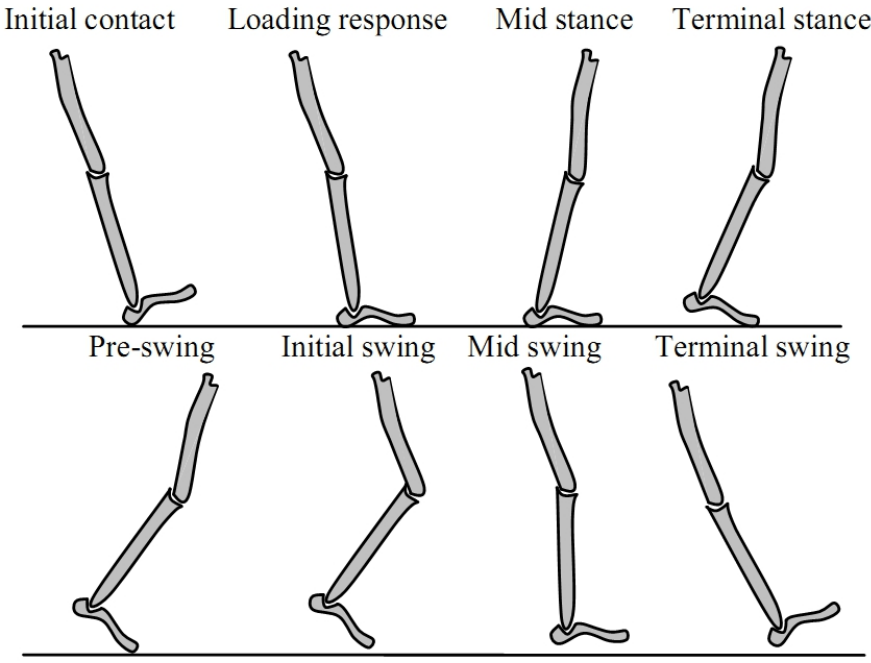

Figure 8: Gait phase of normal walking [63]

at realtime. The former measures the acceleration of the arm with accelerometer sensors and the latter eliminates the errors whose Euclidean distance is larger than a threshold. Both works described in [63] and [64] use wired sensor networks which may limit the patient's activity and reduce user's level of comfort.

Choquette et al. [65] investigated the feasibility and accuracy of wireless sensor networks in measuring the active time in rehabilitation (ie: the time during which the patient is active physically). They used three Micaz modules with 3D accelerometer sensors that sample and record data at $50 \mathrm{~Hz}$. Collected signals from different modules and axes are combined, low-pass filtered, rectified and high-pass filtered. Finally, the active time is estimated by extracting temporal density of the treated acceleration signals.

The Bio-WWS system described in [66] tracks patient's movement and provides real-time feedback to correct posture and balance. It includes three modules with accelerometer sensors, a PDA acting as a gateway and a lightweight headphone for audio feedback generation. Bio-WWS collects gravitational and dynamic planar accelerations from sensors and computes the sway of the user's center of mass. In addition, it implements an algorithm to convert accelerations into a dynamic sound that guides the user to remain inside a target region. The dynamics of the generated sound (direction, volume, frequency) depends on the user's acceleration and the distance between him and a target region which is very useful for the rehabilitation.

In [22], Olivares et al. developed a WSN to monitor human movement in tele-rehabilitation applications with realtime 3D animation of the movement being performed by the patient. The system is based on wireless sensor nodes called Wagyromag (Wireless Accelerometer GYROscope and MAGnetometer) as well as a calibration and a signal processing algorithms to extract human kinematics from collected data. The Wagyromag sensor measured tilt angles instead of acceleration, angular velocity and magnetic field values. Authors used an accelerometer-aided calibration for the gyroscope calibration and an ellipsoid-fitting calibration for the magnetometer. In order to measure the inclination of body parts with high accuracy, authors developed a sensor fusion and an adaptive filtering approach. This approach used different variation of the Last Mean Square (LMS) and Recursive Least Square (RLS) filters.

In [67], authors proposed a sensor network to monitor the upper limb motion for a home based rehabilitation system. The motion tracking system consists of two sensor nodes (with accelerometer, gyroscope and magnetometer sensors) placed near the wrist and elbow to compute the wrist and elbow joints. In addition, authors developed a Langrangian based optimization technique which integrates data measured by the two sensor nodes to estimate the position of the shoulder joint. The novelty of this work is obtaining the position of three joints (wrist, elbow and shoulder) by using only two sensors.

\subsection{Framework and architectures design}

Much effort has been done in designing easily deployable wireless sensor networks for rehabilitation monitoring. These solutions are based on frameworks that support dynamic network construction and configuration, services discovery, resources discovery and sensor node management at runtime. A combination of different technologies like video sensing, gaming and virtual reality have also been investigated in order to enhance the context awareness and attractiveness of existing solutions.

Mercury [68] is a wearable wireless sensor network platform for motion analysis of patients who suffer from neuromotor disorders. It is based on a flexible programming interface allowing clinicians to implement different policies following the application requirements. This interface enable realtime configuration of sensor sampling rate, storage mode, feature extraction and data transmission mode. Mercury achieves a network lifetime 
Table 5: Comparison of signal processing projects

\begin{tabular}{|c|c|c|c|c|c|c|}
\hline Ref & Objective & Real time & Calibration & Error reduction & Filtering & Fusion \\
\hline [63] & -Detection of 8 gait phases & $x$ & $\sqrt{ }$ & $\sqrt{ }$ & $\checkmark$ & $\sqrt{ }$ \\
\hline [64] & $-3 \mathrm{D}$ arm movement tracking & $\sqrt{ }$ & $x$ & $\checkmark$ & $\checkmark$ & $x$ \\
\hline [65] & -Measurement of active time of rehabilitation & $x$ & $x$ & $\checkmark$ & $\checkmark$ & X \\
\hline [66] & $\begin{array}{l}\text {-Estimation of sway of the user's center of mass } \\
\text { and generation of Audio feedback to correct balance }\end{array}$ & $\sqrt{ }$ & $x$ & $x$ & $x$ & $x$ \\
\hline [22] & -Extraction of human kinematics & $\checkmark$ & $\checkmark$ & $\checkmark$ & $\checkmark$ & $\sqrt{ }$ \\
\hline [67] & -Monitoring upper limb motion & $x$ & $\sqrt{ }$ & $\checkmark$ & $\checkmark$ & $\checkmark$ \\
\hline
\end{tabular}

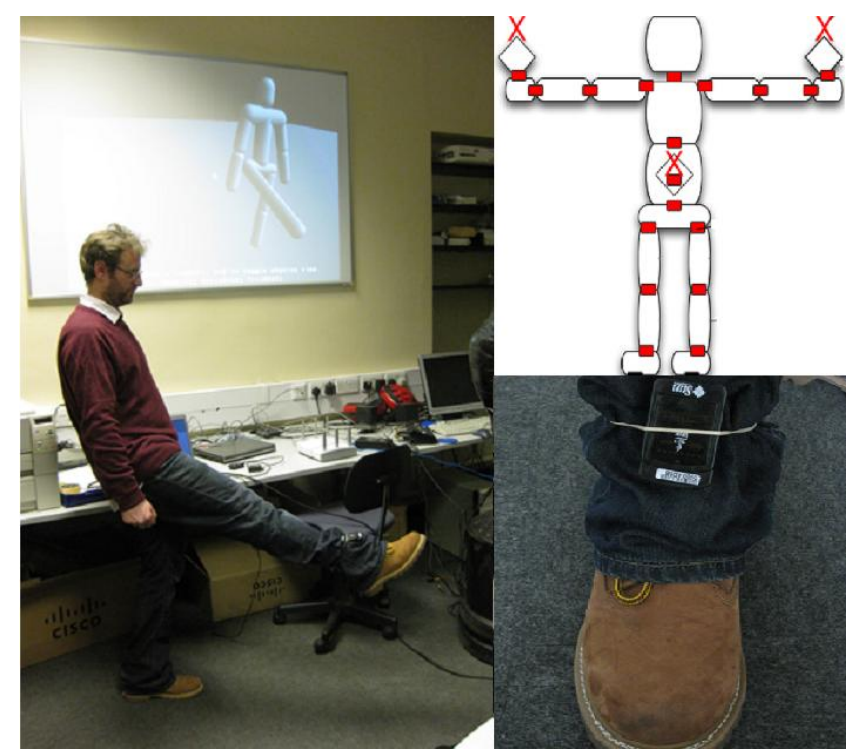

Figure 9: Gaming for rehabilitation [69]

of a couple of days by collecting high-level features from sensor nodes.

Kifayat et al. in [69] presented a framework that combines wireless sensor network technology and gaming to assist rehabilitation of patients with physical disabilities. The system is based on a framework which supports dynamic nodes, sensors and service discovery. The wireless sensor network is attached to the patient's body to measure its acceleration. While the patient plays the game, acceleration data is collected in real-time by the wireless sensor network and sent to a control service allowing the patient to control its virtual representation (avatar showed in figure 9) by physically moving his body. At the end of the game, the framework generates different reports and graphs for doctor and patient.

RehabSPOT proposed in [70] is a highly customizable wireless sensor network platform for rehabilitation built on the top of SunSPOT sensors. The system is based on a software architecture which enables sensors management at runtime (addition/removal of sensors, adjusting sampling rate). In addition, RehabSPOT architecture implements dynamic network construction, devices and sensors discovery and fault tolerance features. Although the usability of RehabSPOT, it requires a long (a fifteen minutes) and a complex setup procedure.

Otto et al. [39] proposed a hardware and software WSN architecture for health monitoring. They developed a prototype made up of two activity sensors ActiS[56], an ECG/tilt sensor (eActis) and a PDA base station. Each sensor node samples sensor signals, analyzes data and transmit application event messages. The software architecture provides several application services such as time synchronization, power management, configuration and commands transmission.

Hamon (Health signs and Activity MONitoring) [28] is a realtime monitoring framework which can be used in several physical rehabilitation such as cardia rehabilitation and Parkinson's Disease rehabilitation. The main objective of this framework is to provide an open and flexible software for the development of applications which collect, analyze and present data collected by a WSN. Hamon can monitor the number of sensor nodes making it easy to collect data from any newly added sensor node. In addition, the framework can implement any processing algorithm for data interpretation.

\section{5. discussion}

There has been a host of research works on WSN for rehabilitation supervision which we reviewed in this section. We classified these works into four categories following their design objective. The first category addressed challenges faced at the hardware level. The objective of this class of works was to build unobtrusive, miniaturized and multimodal sensor nodes fitted to rehabilitation supervision. To achieve their objective, most of researchers designed and implemented new hardware platforms integrating intelligent sensor boards along with different kind of sensors (see table 3). Data collected by this sensors was locally processed before being wirelessly sent to the base station. Unfortunately, these works did not consider energy saving neither the fixation of sensor nodes in their design.

The second category of works investigates the transport of collected data. They proposed communication protocols to wirelessly gathering samples generated by sensors and collecting them at a central gateway. These protocols are often based on a TDMA or an hybrid CSMA/TDMA access technic in order to provide reliable data collection under high data transmission rate (see table 4). However, other QoS mechanisms such as traffic realtime communication, prioritization and service differentiation has not been proposed. In addition, energy efficiency is a 
Table 6: Comparison of rehabilitation frameworks

\begin{tabular}{lllll}
\hline Ref & Node discovery & Sensor discovery & Dynamic configuration & Other features \\
\hline \hline$[68]$ & $X$ & $X$ & $\begin{array}{l}\text {-Sampling rate } \\
\text {-Storage mode } \\
\text {-Transmission mode }\end{array}$ & $\begin{array}{l}\text {-Features extraction } \\
\text {-Energy management }\end{array}$ \\
\hline$[69]$ & $\checkmark$ & $\checkmark$ & -Not supported & -Gaming \\
\hline$[70]$ & $X$ & $X$ & -Sampling rate & -Fault tolerance \\
\hline$[39]$ & $\checkmark$ & $X$ & -Supported but not detailed & $\begin{array}{l}\text {-Energy management } \\
\text {-Visual node identification } \\
\end{array}$ \\
\hline$[28]$ & $\checkmark$ & $X$ & -Not supported & -Realtime alerts and feedback \\
\hline \hline
\end{tabular}

major challenge and a paramount design consideration that has not been considered.

The third category targets the extraction of clinical relevant information from the collected data. This information should evolve into medical knowledge which is useful and viable for doctors. These works proposed efficient signal processing algorithms combining several optimized techniques such as features extraction, fusion and clustering. Most of these works used filtering technics (high-pass filters, low-pass filters, Kalman filters) to clean out the collected data from noise and errors. However, adequate calibration technics, to reduce the undesired components and errors caused by different kind of offsets, should be developed and used. In addition, some proposed algorithms must be optimized to support realtime data processing.

The last category deals with the development of flexible, easy to use and efficient architectures for rehabilitation supervision. Namely, they proposed frameworks which support deployment, configuration and management of nodes in real-time. Combinations with other technologies, such as games and virtual reality, have been proposed and successfully implemented. Table 6 shows different features implemented by works falling in this category.

It's clear from the number of surveyed works that WSN for rehabilitation is a broad and a complex research area. Several research communities are involved in this field proposing complementary innovative solutions. However, these research communities worked solitary, each one separately on her own, to tackle challenges introduced by this new technology. Consequently, there's no complete framework today with a whole design that considers node design, data transmission and data processing for rehabilitation supervision. We believe that bridging researchers with different scientific backgrounds could have a significant impact on the development of such solution and could improve the way rehabilitation is provided today.

\section{Open Issues and Potential Research Directions}

In this section, we describe the main open issues and suggest directions for future researches and works

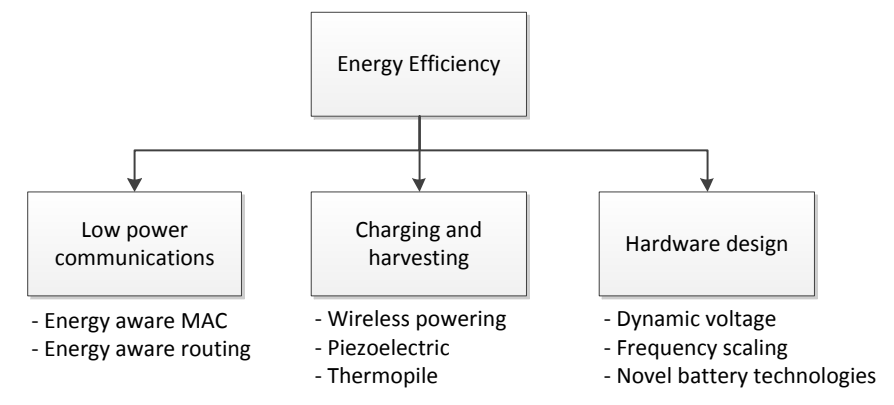

Figure 10: Energy efficiency solution for WSN in rehabilitation

\subsection{Advanced hardware design}

Considerable amount of research has been conducted on the design of unobtrusive and miniaturized sensor nodes. However, a lot of open issues still exist. The level of comfort necessary for widespread adoption of WSN in rehabilitation supervision requires more miniaturization of batteries, circuits, sensors and radio transceivers. Wire connections should be completely removed because they may limit the user activity and significantly interfere with his normal behavior. For instance, developing electrodes with separate radio transceiver to wirelessly connect to an EMG board remains a challenge [16]. Smart textiles [71] is an open research area with promising potential due to good contact between the sensors and the skin [11] leading to steady fixation and reliable motion estimation.

\subsection{Energy saving}

The unobtrusiveness requirement obviously limits the size of the batteries that will power the node and hence reduce the network lifetime. Consequently, we believe that a combination of power-optimal hardware design, energy harvesting techniques and low power communications is the optimal solution for achieving prolonged continuous monitoring (Figure. 10). Activity-aware energy models are an interesting research direction in order to minimize the power consumption. In these models, the node dynamically manages its sensors sampling, data processing and data transmission following the patient activity level [72]. For instance, a context-aware episodic sampling 
technique, such as developed in [73], can be used to reduce the sampling frequency when the patient takes a rest between two rehabilitation exercises. Also, an on-demand performance processor with dynamic voltage and frequency scaling [74] can be used to provide high performance when required and low power consumption otherwise. Developing such on-demand performance processors remains an open research challenge.

Energy harvesting is another option to tackle the energy efficiency challenge, but the amount of power available from such techniques is relatively small. In rehabilitation applications, solar cells can not be used since sensors are often worn under the patient clothes [75]. However, power scavenging methods through motion (Piezoelectric [76]) or body heat (thermopile [77]) have a good potential. These techniques turn the patient ambient energy (motion or thermal gradients generated during exercises) into an electrical form in order to supply power to nodes. However, further research has to be conducted in order to tackle the poor density and poor miniaturization possibilities of these techniques. More extensive discussions on these techniques can be found in [78] and [79].

Development of novel power supplies is paramount to tackle battery recharging issues. Recently, researchers from the University of Illinois developed a novel battery that can be $90 \%$ charged in 2 minutes [80]. Another hot research topic that must be investigated is wireless powering methods such as light, capacitivo or ultra sonic techniques [81][82]. Researchers from MIT [83] succeeded in wirelessly powering sensor nodes over several meters using magnetic resonance. We believe that these kind of technologies can easily alleviate the battery charging issue with elderly or disabled rehabilitation patients.

\section{3. calibration}

Calibration techniques are paramount for the accuracy of measured data especially in dynamic conditions due to patient activity. Design of hardware and software calibration techniques is still an open research area. Particularly, efficient calibration techniques are still needed for sensors other than accelerometers [14]. Self calibration, as proposed by Gietzalt et al in [84], should be investigated to enable a sensor node to automatically compute the offset and adjust his calibration algorithm.

\subsection{Development and evaluation of improved propagation channel models}

Characterization of On-Body channel is a hard and open research challenge due to the complexity of human tissues and body structure. Several propagation patterns has been proposed recently [85][44][86]. However, none of them take into account patient movements which is well known to have a significant impact on communication performance [45]. New models that consider patient activity during rehabilitation are needed. Also, thorough works should be conducted to evaluate existing models at lower layer (antennas performance) as well as at higher layers (energy consumption, network performance, QoS) since their impact on the network performance have not been studied yet [16].

\subsection{Development of new communication technologies}

Wireless transmission suffer from fading because of the body structure and the patient movements during rehabilitation. Hence, we believe that novel communication technologies can improve the network performances. Intra Body Communication technologies (IBC), which use the human body as a communication channel, can be a breakthrough solution for this problem. Galvanic coupling [87] uses two coupler electrodes inducing current into the human tissue and two detector electrodes sensing the potential difference. The signal is transformed into modulated electrical signal which is applied differentially over the two coupler electrodes and generates an electro-magnetic field in the body. The signal is received by the two coupler electrodes and the original signal is extracted. Researchers from NTT (Nippon Telegraph and Telephone) brought a major breakthrough by using Electro-Optic (EO) crystal for IBC and reached a speed of $10 \mathrm{MB} / \mathrm{s}$ half duplex communication through $150 \mathrm{~cm}$ [88]. Bone conduction techniques, such as OsteoConduct [89], is another IBC based on mechanically excited bone conduction inside the human musculoskeletal system. Since these new technologies are still in their infancy, a strong need exists for further research and investigation. Particularly, their impact on human health necessitates further investigation for social acceptance.

\subsection{Bio-effect of radio transmission}

Human exposure to electromagnetic fields generated by sensor nodes during rehabilitation should be kept minimal to avoid advert effect. Some works have investigated the adverse biological effect of radio transmissions on human body in wireless sensor networks [90] but research in this field is still incomplete. IEEE issued a standard which contains recommendations to protect people against harmful effect of electromagnetic fields [91]. This standard defines a threshold which must be respected to guarantee safety of patient in continuous, longterm exposure to electromagnetic fields. According to this, the output transmission of sensor nodes should be kept minimal to cope with health concerns [92]. Low power transmission through small antenna causes high transmission errors. Consequently, antennas need to be controlled by an intelligent coupling circuit to provide both necessary signal strength and safety level which is a complex technical challenge.

\subsection{Quality of Service}

The MAC layer is a vital element for the communication protocols as well as for the whole system design (performance, lifetime). However, only few works on rehabilitation supervision have considered the design of an appropriate communication protocol. Protocols with service differentiation, realtime communications and energy conservation should be proposed like in [93] and [94]. Schedule-based protocols perform well in terms of reliability and energy consumption under high contention conditions of rehabilitation supervision. However, the main drawback of these protocols is that they require tight synchronization. Li and Tan [95] proposed an ingenious synchronization mechanism exploiting human heartbeat rhythm infor- 
mation. By following the rhythm, the network can achieve time synchronization without having to send any message.

\subsection{Routing}

The development of appropriate routing algorithms is of great importance. Indeed, all existing WSN for rehabilitation adopted a star topology with one-hop communications between each node and the gateway. However, there are evidence that multi-hop communications can be better than one hop communications in several cases [48][49]. Thus, multi-hop communications should be used in rehabilitation applications and their performance should be investigated. Specific context aware routing protocols able to tune the network topology following the environment are needed. Furthermore, more appropriate routing protocols such as temperature aware routing (ALTR [96]), cluster based routing (AnyBody [97]) or cross layer routing (CICADA [98]) can be used.

\subsection{Software architecture}

While a lot of researches have been carried out in the design of signal processing algorithms, only few studies have been done regarding data presentation, progress monitoring and performance feedback. Existing WSN require data transformation algorithms in order to present data in high level format. Indeed, a challenge arises from the gap between the low level sensor output and the high level user requirements of therapists [99]. Feedback in real time has not been achieved in all works. In clinical settings, therapist guides the patient to correctly perform useful exercises for his rehabilitation. However, therapist instructions can not be properly acquired in existing solutions. We believe that virtual reality is a potential solution that can guide the patient to perform the correct exercises without therapist intervention. However, the management and interaction with virtual environments raises new open problems discussed in [100]. Interactive video gaming have emerged as new treatment approaches and can brings several improvements to existing solutions. A comprehensive survey on this topic is available in [101]. Finally, Incorporating social dimension such as competitiveness or collaboration should be considered in future works.

\section{Conclusion}

Rehabilitation is a long process undertaken by a patient to return to his initial motor capabilities after a stroke, an accident or a surgery. To achieve optimal rehabilitation outcomes, the patient should be monitored and corrected during exercising. However, continuous monitoring of patients creates management and economic issues that wireless sensor networks can tackle. Indeed, wireless sensor networks for rehabilitation supervision is an emerging interdisciplinary research area that can dramatically improve the way rehabilitation is provided today.

In this paper, we have presented the different applications of wireless sensor networks in rehabilitation as well as their benefits. From the requirements of these applications and the characteristics of WSN, we have identified challenges that should be tackled before fully enjoying the potential of this technology. We have reviewed existing projects conducted by all research communities involved in this field and we have studied their advantages and weakness. Furthermore, we have studied issues that are still open and have proposed possible solutions and directions for future works.

\section{References}

[1] G. Kwakkel, R. van Peppen, R. C. Wagenaar, S. W. Dauphinee, C. Richards, A. Ashburn, K. Miller, N. Lincoln, C. Partridge, I. Wellwood, P. Langhorne, Effects of augmented exercise therapy time after stroke: A Meta-Analysis, Stroke 35 (11).

[2] H. Zhou, H. Hu, Human motion tracking for rehabilitation A survey, Biomed. Signal Process. Control 3 (1) (2008) 1-18.

[3] T. B. Moeslund, E. Granum, A survey of computer vision-based human motion capture, Computer Vision and Image Understanding 81 (3) (2001) 231-268.

[4] D. Gonzalez-Ortega, F. Diaz-Pernas, M. Martinez-Zarzuela, M. AntonRodriguez, J. Diez-Higuera, D. Boto-Giralda, Real-time hands, face and facial features detection and tracking: Application to cognitive rehabilitation tests monitoring, Journal of Network and Computer Applications 33 (4) (2010) 447-466.

[5] [link].

URL http: //www . codamotion. com

[6] [link]. URL http: //www. qualisys. com/

[7] J. Yoon, B. Novandy, C. Yoon, K. Park, A 6-DOF gait rehabilitation robot with upper and lower limb connections that allows walking velocity updates on various terrains, IEEE/ASME Transactions on Mechatronics 15 (2) (2010) 201-215.

[8] A. Duschau-Wicke, J. von Zitzewitz, A. Caprez, L. Lunenburger, R. Riener, Path control: A method for Patient-Cooperative Robot-Aided gait rehabilitation, IEEE Transactions on Neural Systems and Rehabilitation Engineering 18 (1) (2010) 38-48.

[9] W. Y. Wong, M. S. Wong, K. H. Lo, Clinical applications of sensors for human posture and movement analysis: a review, Prosthetics and Orthotics International 31 (1) (2007) 62-75.

[10] A. Hadjidj, A. Bouabdallah, Y. Challal, Rehabilitation supervision using wireless sensor networks, in: IEEE International Symposium on a World of Wireless, Mobile and Multimedia Networks (WoWMoM), IEEE, 2011.

[11] A. Pantelopoulos, N. G. Bourbakis, A survey on wearable Sensor-Based systems for health monitoring and prognosis, IEEE Transactions on Systems, Man, and Cybernetics, Part C: Applications and Reviews 40 (1) (2010) 1-12.

[12] Y. Hao, R. Foster, Wireless body sensor networks for health-monitoring applications, Physiological Measurement 29 (11) (2008) 27-56.

[13] J. Ko, C. Lu, M. B. Srivastava, J. A. Stankovic, A. Terzis, M. Welsh, Wireless sensor networks for healthcare, Proceedings of the IEEE 98 (11) (2010) 1947-1960.

[14] H. Alemdar, C. Ersoy, Wireless sensor networks for healthcare: A survey, Computer Networks 54 (15) (2010) 2688-2710.

[15] S. Patel, H. Park, P. Bonato, L. Chan, M. Rodgers, A review of wearable sensors and systems with application in rehabilitation, Journal of neuroengineering and rehabilitation 9 (2012) 21.

[16] M. Chen, S. Gonzalez, A. Vasilakos, H. Cao, V. C. Leung, Body area networks: A survey, Mobile Networks and Applications 16 (2) (2011) 171-193.

[17] H. Cao, V. Leung, C. Chow, H. Chan, Enabling technologies for wireless body area networks: A survey and outlook, IEEE Communications Magazine 47 (12) (2009) 84-93.

[18] B. Latr, B. Braem, I. Moerman, C. Blondia, P. Demeester, A survey on wireless body area networks, Wireless Networks 17 (1) (2011) 1-18.

[19] S. Ullah, H. Higgins, B. Braem, B. Latre, C. Blondia, I. Moerman, S. Saleem, Z. Rahman, K. S. Kwak, A comprehensive survey of wireless body area networks, Journal of Medical Systems.

[20] Y. Jung, D. Kang, J. Kim, Upper body motion tracking with iner- 
tial sensors, in: 2010 IEEE International Conference on Robotics and Biomimetics (ROBIO), Tianjin, China, 2010, pp. 1746-1751.

[21] R. Perez, U. Costa, M. Torrent, J. Solana, E. Opisso, C. Caceres, J. M. Tormos, J. Medina, E. J. Gomez, Upper limb portable motion analysis system based on inertial technology for neurorehabilitation purposes, Sensors 10 (12) (2010) 10733-10751.

[22] A. Olivares, G. Olivares, F. Mula, J. Gorriz, J. Ramirez, Wagyromag: Wireless sensor network for monitoring and processing human body movement in healthcare applications, Journal of Systems Architecture (0).

[23] M. Soini, J. Nummela, P. Oksa, L. Ukkonen, L. Sydanheimo, Wireless body area network for hip rehabilitation system, Ubiquitous Computing and Communication Journal 3 (2008) 7.

[24] M. A. Hanson, H. C. Powell, A. T. Barth, J. Lach, M. Brandt-Pearce, Neural network gait classification for On-Body inertial sensors, in: Sixth International Workshop on Wearable and Implantable Body Sensor Networks, Berkeley, USA, 2009, pp. 181-186.

[25] H. Ying, M. Schlosser, A. Schnitzer, T. Schafer, M. E. Schlafke, S. Leonhardt, M. Schiek, Distributed intelligent sensor network for the rehabilitation of parkinson's patients, IEEE Trans. Inf. Technol. Biomed. 15 (2) (2011) 268-276

[26] W. H. Press, S. A. Teukolsky, W. T. Vetterling, B. P. Flannery, Numerical Recipes in C, Cambridge, 1992.

[27] A. R. Guraliuc, A. A. Serra, P. Nepa, G. Manara, F. Potorti, Detection and classification of human arm movements for physical rehabilitation, in: 2010 IEEE Antennas and Propagation Society International Symposium (APSURSI), Pisa, Italy, 2010, pp. 1-4.

[28] M. F. Alhamid, J. Saboune, A. Alamri, A. El Saddik, Hamon: An activity recognition framework for health monitoring support at home, in: 2011 IEEE Instrumentation and Measurement Technology Conference (I2MTC), Binjiang, 2011, pp. 1-5.

[29] C. C. Holmes, N. M. Adams, A probabilistic nearest neighbour method for statistical pattern recognition, Journal Of The Royal Statistical Society Series B 64 (2) (2002) 295-306.

[30] [link].

URL http://www.cs.ubc.ca/ murphyk/Bayes/bnintro.html

[31] B. P. Jarochowski, S. Shin, D. Ryu, H. Kim, Ubiquitous rehabilitation center: An implementation of a wireless sensor network based rehabilitation management system, in: International Conference on Convergence Information Technology, 2007, Gyeongju, Korea, 2007, pp. 2349_ 2358.

[32] J. Bravo, F. J. Navarro, J. Fontecha, R. Hervas, A mobile proposal for frailty monitoring by rehabilitation and physical daily activity, in: 2011 IEEE International Conference on Consumer Electronics - Berlin (ICCE-Berlin), Berlin, Germany, 2011, pp. 176-180.

[33] A. Cappozzo, U. Della Croce, A. Leardini, L. Chiari, Human movemen analysis using stereophotogrammetry: Part 1: theoretical background, Gait \& Posture 21 (2) (2005) 186-196

[34] G. C. Burdea, D. Cioi, J. Martin, D. Fensterheim, M. Holenski, The rutgers arm II rehabilitation System feasibility study, IEEE Transactions on Neural Systems and Rehabilitation Engineering 18 (5) (2010) 505514.

[35] M. S. Cameirao, S. B. I. Badia, E. D. Oller, P. F. M. J. Verschure, Neurorehabilitation using the virtual reality based rehabilitation gaming system: methodology, design, psychometrics, usability and validation, J. Neuroeng Rehabil. 7 (2010) 48.

[36] A. Alamri, J. Cha, A. El Saddik, AR-REHAB: an augmented reality framework for Poststroke-Patient rehabilitation, IEEE Trans. Instrum. Meas. 59 (10) (2010) 2554-2563.

[37] P. Kulkarni, Y. Ozturk, mPHASiS: mobile patient healthcare and sensor information system, Journal of Network and Computer Applications 34 (1) (2011) 402-417.

[38] U. Anliker, J. Beutel, M. Dyer, R. Enzler, P. Lukowicz, L. Thiele, G. Troster, A systematic approach to the design of distributed wearable systems, IEEE Trans. Comput. 53 (8) (2004) 1017- 1033.

[39] C. Otto, A. Milenkovic, C. Sanders, E. Jovanov, System architecture of a wireless body area sensor network for ubiquitous health monitoring, J. Mob. Multi. 1 (4) (2006) 307-326.

[40] M. A. Lafortune, Three-dimensional acceleration of the tibia during walking and running, Journal of Biomechanics 24 (10) (1991) 877-886.

[41] L. Light, G. McLellan, L. Klenerman, Skeletal transients on heel strike in normal walking with different footwear, Journal of Biomechanics 13 (6) (1980) 477-480

[42] H. Harms, O. Amft, G. Troster, Influence of a loose-fitting sensing garment on posture recognition in rehabilitation, in: IEEE Biomedical Circuits and Systems Conference, 2008. BioCAS 2008, 2008, pp. 353-356.

[43] S. J. Marinkovic, E. M. Popovici, C. Spagnol, S. Faul, W. P. Marnane, Energy-Efficient low duty cycle MAC protocol for wireless body area networks, IEEE Trans. Inf. Technol. Biomed. 13 (6) (2009) 915-925.

[44] A. Taparugssanagorn, A. Rabbachin, M. Hamalainen, J. Saloranta, J. Iinatti, A review of channel modelling for wireless body area network in wireless medical communications, in: 11th International Symposium on Wireless Personal Multimedia Communications, Lapland, Finand, 2008.

[45] F. Di Franco, C. Tachtatzis, B. Graham, M. Bykowski, D. C. Tracey, N. F. Timmons, J. Morrison, The effect of body shape and gender on wireless body area network on-body channels, in: 2010 IEEE Middle East Conference on Antennas and Propagation (MECAP), Cairo, Egypt, 2010, pp. 1-3

[46] A. Hadjidj, Y. Challal, R. Li, Toward a High-Fidelity wireless sensor network for rehabilitation supervision, in: IEEE 36th Conference on Local Computer Networks (LCN), Bonn, Germany, 2011

[47] T. Zasowski, F. Althaus, M. Stager, A. Wittneben, G. Troster, UWB for noninvasive wireless body area networks: channel measurements and results, in: 2003 IEEE Conference on Ultra Wideband Systems and Technologies, Virginia, USA, 2003, pp. 285- 289.

[48] A. Natarajan, B. de Silva, K. Yap, M. Motani, To hop or not to hop: Network architecture for body sensor networks, in: SECON '09, Rome, Italy, 2009, pp. 1-9.

[49] A. Natarajan, M. Motani, B. de Silva, K. Yap, K. C. Chua, Investigating network architectures for body sensor networks, in: HealthNet 07, New York, NY, USA, 2007, pp. 19-24.

[50] J. J. Kavanagh, H. B. Menz, Accelerometry: a technique for quantifying movement patterns during walking, Gait \& Posture 28 (1) (2008) 1-15.

[51] H. Sveistrup, Motor rehabilitation using virtual reality, Journal of NeuroEngineering and Rehabilitation 1 (1) (2004) 10

[52] C. Bryanton, J. Bosse, M. Brien, J. Mclean, A. McCormick, H. Sveistrup, Feasibility, motivation, and selective motor control: Virtual reality compared to conventional home exercise in children with cerebral palsy, CyberPsychology \& Behavior 9 (2) (2006) 123-128.

[53] H. Saito, T. Watanabe, A. Arifin, Ankle and knee joint angle measurements during gait with wearable sensor system for rehabilitation, in: World Congress on Medical Physics and Biomedical Engineering, September 7-12, 2009, Munich, Germany, 2009, p. 506509.

[54] C. K. Lim, I. Chen, Z. Luo, S. H. Yeo, A low cost wearable wireless sensing system for upper limb home rehabilitation, in: 2010 IEEE Conference on Robotics, Automation and Mechatronics, Singapore, 2010, pp. 1-8.

[55] A. Milenkovic, C. Otto, E. Jovanov, Wireless sensor networks for personal health monitoring: Issues and an implementation, Computer Communications 29 (13) (2006) 2521-2533.

[56] E. Jovanov, A. Milenkovic, C. Otto, P. de Groen, A wireless body area network of intelligent motion sensors for computer assisted physical rehabilitation, Journal of NeuroEngineering and Rehabilitation 2 (1) (2005) 6.

[57] H. Ying, M. Scholosser, A. Schnitzer, S. Leonhardt, M. Schiek, Distributed intelligent sensor network for neurological rehabilitation research, in: 4th European Conference of the International Federation for Medical and Biological Engineering, 2009, pp. 1714-1717.

[58] M. Schloesser, A. Schnitzer, H. Ying, C. Silex, M. Schiek, iSANLA: intelligent sensor and actuator network for life science applications, in: EMBC 2008, 2008, pp. 2369-2372.

[59] M. Hamel, R. Fontaine, P. Boissy, In-home telerehabilitation for geriatric patients, Engineering in Medicine and Biology Magazine, IEEE 27 (4) (2008) 29-37.

[60] [link]. URL http: //www. thisisant.com/

[61] J. A. Afonso, H. D. Silva, P. Macedo, L. A. Rocha, Design and implementation of a wireless sensor network applied to motion capture, in: Portuguese conference on wireless sensor networks, Coimbra, Portugal, 2011.

[62] J. A. Afonso, L. A. Rocha, H. R. Silva, J. H. Correia, MAC protocol for 
Low-Power Real-Time wireless sensing and actuation, in: 13th IEEE International Conference on Electronics, Circuits and Systems, 2006. ICECS '06, Nice, France, 2006, pp. 1248-1251.

[63] T. Liu, Y. Inoue, K. Shibata, R. Zheng, Measurement of human lower limb orientations and ground reaction forces using wearable sensor systems, in: IEEE/ASME international conference on Advanced intelligent mechatronics, 2007, p. 16.

[64] H. Zhou, H. Hu, N. Harris, Application of wearable inertial sensors in stroke rehabilitation, in: EMBC 2006, 2006, pp. 6825-6828.

[65] S. Choquette, M. Hamel, P. Boissy, Accelerometer-based wireless body area network to estimate intensity of therapy in post-acute rehabilitation, Journal of NeuroEngineering and Rehabilitation 5 (1) (2008) 20.

[66] D. Brunelli, E. Farella, L. Rocchi, M. Dozza, L. Chiari, L. Benini, Biofeedback system for rehabilitation based on a wireless body area network, in: Fourth Annual IEEE International Conference on Pervasive Computing and Communications Workshops (PERCOMW'06), Pisa, Italy, 2006, pp. 527-531.

[67] H. Zhou, T. Stone, H. Hu, N. Harris, Use of multiple wearable inertial sensors in upper limb motion tracking, Medical Engineering \& Physics 30 (1) (2008) 123-133.

[68] K. Lorincz, B. Chen, G. W. Challen, A. R. Chowdhury, S. Patel, P. Bonato, M. Welsh, Mercury: A wearable sensor network platform for highfidelity motion analysis, in: 7th Conference on Embedded Networked Sensor Systems, 2009, pp. 183-196.

[69] K. Kifayat, P. Fergus, S. Cooper, M. Merabti, Body area networks for movement analysis in physiotherapy treatments, in: 24th IEEE International Conference on Advanced Information Networking and Applications Workshops, 2010, pp. 866-872.

[70] M. Zhang, A. A. Sawchuk, A customizable framework of body area sensor network for rehabilitation, in: 2nd International Symposium on Applied Sciences in Biomedical and Communication Technologies, Bratislava, Slovakia, 2009, pp. 1-6.

[71] S. Park, S. Jayaraman, Smart Textile-Based wearable biomedical systems: A transition plan for research to reality, IEEE Trans. Inf. Technol. Biomed. 14 (1) (2010) 86-92.

[72] F. Sun, C. Kuo, M. Griss, PEAR: power efficiency through activity recognition (for ECG-based sensing), in: 2011 5th International Conference on Pervasive Computing Technologies for Healthcare (PervasiveHealth), Dublin, Ireland, 2011, pp. 115-122.

[73] L. K. Au, M. A. Batalin, T. Stathopoulos, A. A. Bui, W. J. Kaiser, Episodic sampling: Towards energy-efficient patient monitoring with wearable sensors, in: Annual International Conference of the IEEE Engineering in Medicine and Biology Society, 2009. EMBC 2009, Minneapolis, USA, 2009, pp. 6901-6905.

[74] D. Raskovic, D. Giessel, Dynamic voltage and frequency scaling for OnDemand performance and availability of biomedical embedded systems, IEEE Trans. Inf. Technol. Biomed. 13 (6) (2009) 903-909.

[75] N. Tadayon, S. Khoshroo, E. Askari, H. Wang, H. Michel, Power management in SMAC-based energy-harvesting wireless sensor networks using queuing analysis, Journal of Network and Computer Applications (0). doi:10.1016/j.jnca.2012.01.009.

[76] A. Olivares, G. Olivares, J. Gorriz, J. Ramirez, A study of vibrationbased energy harvesting in activities of daily living, in: 4th International Conference on Pervasive Computing Technologies for Healthcare (PervasiveHealth), 2010, pp. $1-4$.

[77] D. C. Hoang, Y. K. Tan, H. B. Chng, S. K. Panda, Thermal energy harvesting from human warmth for wireless body area network in medical healthcare system, in: International Conference on Power Electronics and Drive Systems, 2009, pp. 1277-1282.

[78] P. D. Mitcheson, Energy harvesting for human wearable and implantable bio-sensors, in: EMBC 2010, 2010, pp. 3432-3436.

[79] E. Romero, R. O. Warrington, M. R. Neuman, Energy scavenging sources for biomedical sensors, Physiological Measurement 30 (9) (2009) 35-62.

[80] H. Zhang, X. Yu, P. V. Braun, Three-dimensional bicontinuous ultrafastcharge and -discharge bulk battery electrodes, Nature Nanotechnology 6 (5) (2011) 277-281.

[81] F. Zhang, X. Liu, S. A. Hackworth, R. J. Sclabassi, M. Sun, In vitro and in vivo studies on wireless powering of medical sensors and implantable devices, in: Life Science Systems and Applications Workshop, 2009. LiSSA 2009. IEEE/NIH, 2009, pp. 84-87.
[82] F. Zhang, S. A. Hackwoth, X. Liu, C. Li, M. Sun, Wireless power delivery for wearable sensors and implants in body sensor networks, in: EMBC 2010, 2010, pp. 692-695.

[83] A. Kurs, A. Karalis, R. Moffatt, J. D. Joannopoulos, P. Fisher, M. Soljacic, Wireless power transfer via strongly coupled magnetic resonances, Science 317 (5834) (2007) 83 -86.

[84] M. Gietzelt, K. H. Wolf, M. Marschollek, R. Haux, Automatic selfcalibration of body worn triaxial-accelerometers for application in healthcare, in: Second International Conference on Pervasive Computing Technologies for Healthcare, PervasiveHealth 2008, Tampere, Finland, 2008, pp. 177-180.

[85] T. Alves, B. Poussot, J. M. Laheurte, Analytical propagation modeling of BAN channels based on the Creeping-Wave theory, IEEE Trans. on Antennas and Propagation 59 (4) (2011) 1269-1274.

[86] A. M. Eid, J. W. Wallace, Accurate modeling of body area network channels using Surface-Based method of moments, IEEE Transactions on Antennas and Propagation 59 (8) (2011) 3022-3030.

[87] M. S. Wegmueller, M. Oberle, N. Felber, N. Kuster, W. Fichtner, Signal transmission by galvanic coupling through the human body, IEEE Trans. Instrum. Meas. 59 (4) (2010) 963-969.

[88] A. Sasaki, M. Shinagawa, K. Ochiai, Principles and demonstration of intrabody communication with a sensitive electrooptic sensor, IEEE Trans. Instrum. Meas. 58 (2) (2009) 457-466.

[89] L. Zhong, D. El-Daye, B. Kaufman, N. Tobaoda, T. Mohamed, M. Liebschner, OsteoConduct: wireless body-area communication based on bone conduction, in: Proceedings of the ICST 2nd international conference on Body area networks, Brussels, Belgium, 2007.

[90] H. Ren, Q. H. M. Max, Bioeffects control in wireless biomedical sensor networks, in: 3rd Annual IEEE Communications Society on Sensor and Ad Hoc Communications and Networks, 2006. SECON '06, Vol. 3 , 2006, pp. 896-904.

[91] Ieee standard for safety levels with respect to human exposure to radio frequency electromagnetic fields, $3 \mathrm{khz}$ to $300 \mathrm{ghz}$, IEEE Std C95.12005 (Revision of IEEE Std C95.1-1991) (2006) 1-238.

[92] H. Ren, M. Q. H. Meng, Rate control to reduce bioeffects in wireless biomedical sensor networks, in: 3rd Annual International Conference on Mobile and Ubiquitous Systems - Workshops, 2006, San Jose, California, USA, 2006, pp. 1-7.

[93] N. Saxena, A. Roy, J. Shin, Dynamic duty cycle and adaptive contention window based qos-mac protocol for wireless multimedia sensor networks, Computer Networks 52 (2008) 2532-2542.

[94] B. Otal, L. Alonso, C. Verikoukis, Highly reliable energy-saving mac for wireless body sensor networks in healthcare systems, IEEE J. Sel. Areas Commun. 27 (2009) 553-565.

[95] H. Li, J. Tan, Heartbeat driven medium access control for body sensor networks, in: HealthNet 07, NY, USA, 2007, pp. 25-30.

[96] A. Bag, M. Bassiouni, Energy efficient thermal aware routing algorithms for embedded biomedical sensor networks, in: IEEE International Conference on Mobile Adhoc and Sensor Systems (MASS), Vancouver, 2006, pp. 604-609.

[97] T. Watteyne, I. Auge-Blum, M. Dohler, D. Barthel, AnyBody: a selforganization protocol for body area networks, in: Proceedings of the ICST 2nd international conference on BANs, 2007, pp. 1-7.

[98] B. Latre, B. Braem, I. Moerman, C. Blondia, E. Reusens, W. Joseph, P. Demeester, A low-delay protocol for multihop wireless body area networks, in: Fourth Annual International Conference on Mobile and Ubiquitous Systems: Networking \& Services, MobiQuitous, Philadelphia, USA, 2007, pp. 1-8.

[99] M. Roantree, J. Shi, P. Cappellari, M. F. O’Connor, M. Whelan, N. Moyna, Data transformation and query management in personal health sensor networks, Journal of Network and Computer Applications 35 (4) (2012) 1191-1202.

[100] J. Ibanez, C. Delgado-Mata, Lessons from research on interaction with virtual environments, Journal of Network and Computer Applications 34 (1) (2011) 268-281.

[101] P. Rego, P. M. Moreira, L. P. Reis, Serious games for rehabilitation: A survey and a classification towards a taxonomy, in: 2010 5th Iberian Conference on Information Systems and Technologies (CISTI), Santiago de Compostela, Spain, 2010, pp. 1-6. 